\title{
A QUANTUM COMPUTATIONAL SEMANTICS FOR EPISTEMIC LOGICAL OPERATORS. PART II: SEMANTICS
}

\author{
ENRICO BELTRAMETTI, MARIA LUISA DALLA CHIARA, ROBERTO GIUNTINI, \\ ROBERTO LEPORINI, AND GIUSEPPE SERGIOLI
}

\begin{abstract}
By using the abstract structures investigated in the first Part of this article, we develop a semantics for an epistemic language, which expresses sentences like "Alice knows that Bob does not understand that $\pi$ is irrational". One is dealing with a holistic form of quantum computational semantics, where entanglement plays a fundamental role; thus, the meaning of a global expression determines the contextual meanings of its parts, but generally not the other way around. The epistemic situations represented in this semantics seem to reflect some characteristic limitations of the real processes of acquiring information. Since knowledge is not generally closed under logical consequence, the unpleasant phenomenon of logical omniscience is here avoided.
\end{abstract}

\section{The ePistemic QuAntum COMPUtational Syntax}

The structures, investigated in the first Part of this article, provide the mathematical basis for the development of our epistemic semantics. The basic intuitive idea can be sketched as follows: pieces of quantum information (qumixes) can be denoted by the sentences of a formal language, whose logical connectives correspond to some quantum gates, while the two fundamental epistemic operators (to understand and to know) are interpreted as epistemic operations living in semantic models based on convenient epistemic quantum computational structures. Accordingly, sentences like "At time $t$ Alice knows that Bob does not understand that $\pi$ is irrational" turn out to denote particular examples of qumixes, representing possible states of quantum objects. Let us first introduce the epistemic language that will be used. This language, indicated by $\mathcal{L}^{E p Q C}$, contains atomic sentences (say, "the spin-value in the $x$-direction is up"), including two privileged sentences $\mathbf{t}$ and $\mathbf{f}$ that represent the truth-values Truth and Falsity, respectively. We will use $\mathbf{q}, \mathbf{r}, \ldots$ as metavariables for atomic sentences, and $\alpha, \beta, \ldots$ as metavariables for sentences. The quantum computational connectives of $\mathcal{L}^{E p Q C}$ are: the negation $\neg$ (which corresponds to the gate Negation), the squareroot of the identity $\sqrt{i d}$ (which corresponds to Hadamard), a ternary connective $\mathrm{T}$ (which corresponds to the Toffoli-gate), the exclusive disjunction $\biguplus$ (which corresponds to XOR). The epistemic sub-language of $\mathcal{L}^{E p Q C}$ contains: a finite set of names for epistemic agents $(\mathbf{a}, \mathbf{b}, \ldots)$; a set of names 
$\left(t_{1}, t_{2}, \ldots\right)$ for the elements of a given time-sequence; the epistemic operators $\mathcal{U}$ (to understand) and $\mathcal{K}$ (to know).

For any sentences $\alpha, \beta, \gamma$, the expressions $\neg \alpha, \sqrt{i d} \alpha, \mathrm{T}(\alpha, \beta, \gamma), \alpha \biguplus \beta$ are sentences. For any sentence $\alpha$, for any agent-name a (say, Alice), for any time-name $t$, the expressions $\mathcal{U} \mathbf{a}_{t} \alpha$ (at time $t$ agent $\mathbf{a}$ understands the sentence $\alpha$ ) and $\mathcal{K} \mathbf{a}_{t} \alpha$ (at time $t$ agent a knows the sentence $\alpha$ ) are sentences. Notice that nested epistemic operators are allowed: an expression like $\mathcal{K} \mathbf{a}_{t} \neg \mathcal{U} \mathbf{b}_{t} \alpha$ is a well-formed sentence. The connectives $\neg, \sqrt{i d}, \mathbf{T}, \biguplus$ are called gate-connectives. Any subexpression $\mathcal{U} \mathbf{a}_{t}$ or $\mathcal{K} \mathbf{a}_{t}$ of an epistemic sentence will be called an epistemic connective.

We recall that, for any truth-perspective $\mathfrak{T}$, the Toffoli-gate permits one to define a reversible conjunction $\operatorname{AND}_{\mathfrak{T}}$ (for any $\rho \in \mathfrak{D}\left(\mathcal{H}^{(m)}\right)$ and for any $\left.\sigma \in \mathfrak{D}\left(\mathcal{H}^{(n)}\right)\right)$ :

$$
\operatorname{AND}_{\mathfrak{T}}(\rho, \sigma):=\mathfrak{D}_{\mathrm{T}_{\mathfrak{T}}^{(m, n, 1)}}^{\left(\rho \otimes \sigma \otimes{ }^{\mathfrak{T}} P_{0}^{(1)}\right) .}
$$

Accordingly, from a syntactical point of view, it is reasonable to define (metalinguistically) the logical conjunction $\wedge$ as follows (for any sentences $\alpha$ and $\beta)$ :

$$
\alpha \wedge \beta:=\mathbf{T}(\alpha, \beta, \mathbf{f}) .
$$

We will now introduce some syntactical notions that will be used in our semantics.

\section{Definition 1.1.}

- $\alpha$ is called a gate-sentence iff either $\alpha$ is atomic or the principal connective of $\alpha$ is a gate-connective.

- $\alpha$ is called an epistemic sentence iff $\alpha$ has the form $\mathcal{K} \mathbf{a}_{t} \beta$.

Definition 1.2. (The atomic complexity of a sentence)

The atomic complexity At $(\alpha)$ of a sentence $\alpha$ is the number of occurrences of atomic sentences in $\alpha$.

For instance, $\operatorname{At}(\mathbf{T}(\mathbf{q}, \mathbf{q}, \mathbf{f}))=3$. We will also indicate by $\alpha^{(n)}$ a sentence whose atomic complexity is $n$. The notion of atomic complexity plays an important semantic role. As we will see, the meaning of any sentence whose atomic complexity is $n$ shall live in the domain $\mathfrak{D}\left(\mathcal{H}^{(n)}\right)$. For this reason, $\mathcal{H}^{(A t(\alpha))}$ (briefly indicated by $\mathcal{H}^{\alpha}$ ) will be also called the semantic space of $\alpha$.

Any sentence $\alpha$ can be naturally decomposed into its parts, giving rise to a special configuration called the syntactical tree of $\alpha$ (indicated by STree ${ }^{\alpha}$ ).

Roughly, STree ${ }^{\alpha}$ can be represented as a finite sequence of levels:

$$
\begin{gathered}
\text { Level }_{k}(\alpha) \\
\vdots \\
\text { Level }_{1}(\alpha),
\end{gathered}
$$

where: 
- each $\operatorname{Level}_{i}(\alpha)$ (with $\left.1 \leq i \leq k\right)$ is a sequence $\left(\beta_{1}, \ldots, \beta_{m}\right)$ of subformulas of $\alpha$;

- the bottom level Level $_{1}(\alpha)$ is $\alpha$;

- the top level Level $_{k}(\alpha)$ is the sequence $\left(\mathbf{q}_{1}, \ldots, \mathbf{q}_{r}\right)$, where $\mathbf{q}_{1}, \ldots \mathbf{q}_{r}$ are the atomic occurrences in $\alpha$;

- for any $i$ (with $1 \leq i<k$ ), Level $_{i+1}(\alpha)$ is the sequence obtained by dropping the principal gate-connective in all molecular gate-sentences occurring at $\operatorname{Level}_{i}(\alpha)$, by dropping the epistemic connectives $\left(\mathcal{U} \mathbf{a}_{t}\right.$, $\left.\mathcal{K} \mathbf{a}_{t}\right)$ in all epistemic sentences occurring at $\operatorname{Level}_{i}(\alpha)$ and by repeating all the atomic sentences that occur at $\operatorname{Level}_{i}(\alpha)$.

By Height of $\alpha$ (indicated by Height $(\alpha)$ ) we mean the number of levels of the syntactical tree of $\alpha$.

As an example, consider the following sentence:

$$
\alpha=\mathcal{K} \mathbf{a}_{t} \neg(\mathbf{q} \wedge \neg \mathbf{q})=\mathcal{K} \mathbf{a}_{t} \neg(\mathbf{T}(\mathbf{q}, \neg \mathbf{q}, \mathbf{f}))
$$

(say, "At time $t$ Alice knows the non-contradiction principle", instantiated by means of the atomic sentence $\mathbf{q}$ ).

The syntactical tree of $\alpha$ is the following sequence of levels:

$$
\begin{aligned}
& \text { Level }_{5}(\alpha)=(\mathbf{q}, \mathbf{q}, \mathbf{f}) \\
& \text { Level }_{4}(\alpha)=(\mathbf{q}, \neg \mathbf{q} ; \mathbf{f}) \\
& \text { Level }_{3}(\alpha)=(\mathbf{T}(\mathbf{q}, \neg \mathbf{q}, \mathbf{f})) \\
& \text { Level }_{2}(\alpha)=(\neg \mathbf{T}(\mathbf{q}, \neg \mathbf{q}, \mathbf{f})) \\
& \text { Level }_{1}(\alpha)=(\mathcal{K} \mathbf{a} t \neg(\mathbf{T}(\mathbf{q}, \neg \mathbf{q}, \mathbf{f})))
\end{aligned}
$$

Clearly, Height $(\mathbf{T}(\mathbf{q}, \neg \mathbf{q}, \mathbf{f}))=5$.

More precisely, the syntactical tree of a sentence (whose atomic complexity is $r$ ) is defined as follows.

Definition 1.3. (The syntactical tree of $\alpha$ )

The syntactical tree of $\alpha$ is the following sequence of sentence-sequences:

$$
\operatorname{STree}^{\alpha}=\left(\text { Level }_{1}(\alpha), \ldots, \text { Level }_{k}(\alpha)\right)
$$

where:

- $\operatorname{Level}_{1}(\alpha)=\alpha$;

- Level $i+1$ is defined as follows for any $i$ such that $1 \leq i<k$. The following cases are possible:

(1) Level $_{i}(\alpha)$ does not contain any connective. Hence, Level $(\alpha)=$ $\left(\mathbf{q}_{1}, \ldots, \mathbf{q}_{r}\right)$ and $\operatorname{Height}(\alpha)=i$

(2) Level $_{i}(\alpha)=\left(\beta_{1}, \ldots, \beta_{m}\right)$, and for at least one $j, \beta_{j}$ has a (principal) connective. Consider the following sequence of sentencesequences:

$$
\int_{1}^{\prime}, \ldots, \int_{m}^{\prime}
$$


where: $\int_{h}^{\prime}=\left\{\begin{array}{l}\left(\beta_{h}\right), \text { if } \beta_{h} \text { is atomic; } \\ \int_{h}^{*}, \text { otherwise. Where: } \int_{h}^{*}=\left\{\begin{array}{l}(\delta), \text { if } \beta_{h}=\neg \delta \text { or } \beta_{h}=\sqrt{i d} \delta ; \\ (\gamma, \delta, \theta), \text { if } \beta_{h}=\mathrm{T}(\gamma, \delta, \theta) ; \\ (\gamma, \delta), \text { if } \beta_{h}=\gamma \biguplus \delta ; \\ (\delta), \text { if } \beta_{h}=\mathcal{U} \mathbf{a}_{t} \delta \text { or } \beta_{h}=\mathcal{K} \mathbf{a}_{t} \delta .\end{array}\right.\end{array}\right.$

Then,

$$
\text { Level }_{i+1}(\alpha)=\int_{1}^{\prime} \bullet \ldots \bullet \int_{m}^{\prime}
$$

where $\bullet$ represents the sequence-composition.

\section{The ePistemic quantum Computational SEmantics}

We will now give the basic definitions of our semantics. We will apply to epistemic situations a holistic version of quantum computational semantics (which has been naturally inspired by the characteristic holistic features of the quantum theoretic formalism 1 ). In this semantics any model assigns to any sentence a global meaning that determines the contextual meanings of all its parts (from the whole to the parts!). It may happen that one and the same sentence receives different meanings in different contexts.

Before defining holistic models, we will first introduce the weaker notion of quasi-model of the language $\mathcal{L}^{E p Q C}$.

Definition 2.1. (Quasi-model)

$A$ quasi-model of the language $\mathcal{L}^{E p Q C}$ is a system

$$
\mathcal{M}^{q}=(T, A g, \mathbf{E p S i t}, \text { den })
$$

where:

(1) (T, Ag, EpSit) is an epistemic quantum computational structure2;

(2) den is a function that interprets the individual names of the language. By simplicity, we put:

$$
\operatorname{den}(\mathbf{a})=\mathfrak{a} ; \operatorname{den}(t)=\mathfrak{t} .
$$

Apparently, quasi-models represent partial interpretations of the language: while names of times and of agents receive an interpretation in the framework of a given epistemic situation, meanings of sentences are not determined.

In the first Part of this article we have seen that knowledge operations cannot be generally represented as qumix gates. At the same time, once fixed an epistemic quantum computational structure $\mathcal{S}=(T, A g, \mathbf{E p S i t})$, one can naturally define the following notion of pseudo-gate with respect to $\mathcal{S}$.

Definition 2.2. (Pseudo-gate)

\footnotetext{
${ }^{1}$ See [1] and [3].

${ }^{2}$ See Section 3 of the first Part of this article.
} 
Let $\mathcal{S}=(T, A g, \mathbf{E p S i t})$ be an epistemic quantum computational structure. $A$ pseudo-gate of $\mathcal{S}$ is a operator-product

$$
\mathbf{X}_{1}^{\left(n_{1}\right)} \otimes \ldots \otimes \mathbf{X}_{m}^{\left(n_{m}\right)},
$$

where any $\mathbf{X}_{i}^{\left(n_{i}\right)}$ (with $1 \leq i \leq m$ ) is either a qumix-gate ${ }^{\mathfrak{D}} G_{\mathfrak{T}}^{\left(n_{i}\right)}$ with respect to a truth-perspective $\mathfrak{T}$ or an epistemic operation $\left(\mathbf{U}_{\mathfrak{a}_{\mathfrak{t}}}^{\left(n_{i}\right)}\right.$ or $\mathbf{K}_{\mathfrak{a}_{\mathfrak{t}}}^{\left(n_{i}\right)}$ ) of $\mathcal{S}$.

One can show that for any choice of a truth-perspective $\mathfrak{T}$ and of a quasi-model $\mathcal{M}^{q}=(T, A g, \mathbf{E p S i t}$, den $)$, the syntactical tree of a sentence $\alpha$ uniquely determines a sequence of pseudo-gates, that will be called the $\left(\mathfrak{T}, \mathcal{M}^{q}\right)$-pseudo-gate tree of $\alpha$.

As an example, consider again the sentence

$$
\alpha=\mathcal{K} \mathbf{a}_{\mathbf{t}} \neg(\mathbf{q} \wedge \neg \mathbf{q})=\mathcal{K} \mathbf{a}_{\mathbf{t}} \neg(\mathbf{T}(\mathbf{q}, \neg \mathbf{q}, \mathbf{f}))
$$

and its syntactical tree.

Apparently, $\operatorname{Level}_{4}(\alpha)$ is obtained from $\operatorname{Level}_{5}(\alpha)$ by repeating the first occurrence of $\mathbf{q}$, by negating the second occurrence of $\mathbf{q}$ and by repeating f. Hence the pseudo-gate that corresponds to $\operatorname{Level}_{4}(\alpha)$ will be ${ }^{\mathfrak{D}} \mathrm{I}^{(1)} \otimes$

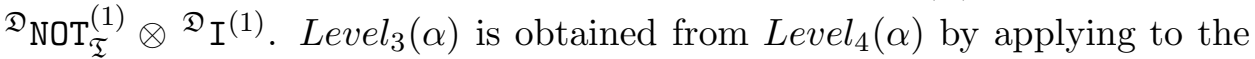
three sentences occurring at $\operatorname{Level}_{4}(\alpha)$ the connective T. Hence the pseudogate that corresponds to Level $_{3}(\alpha)$ will be ${ }^{\mathfrak{D}} \mathrm{T}_{\mathfrak{T}}^{(1,1,1)}$. Level $2(\alpha)$ is obtained from $\operatorname{Level}_{3}(\alpha)$ by applying to the sentence occurring at $\operatorname{Level}_{3}(\alpha)$ the connective $\neg$. Hence the pseudo-gate that corresponds to $\operatorname{Level}_{2}(\alpha)$ will be $\mathfrak{D}_{\mathrm{NOT}_{\mathfrak{T}}^{(3)} \text {. Finally, Level }}(\alpha)$ is obtained from $\operatorname{Level}_{2}(\alpha)$ by applying to the sentence occurring at Level $_{2}(\alpha)$ the epistemic connective $\mathcal{K} \mathbf{a}_{t}$. Hence the pseudo-gate that corresponds to Level $_{1}(\alpha)$ will be $\mathbf{K}_{\mathfrak{a}_{\mathfrak{t}}}^{(3)}$.

On this basis, the $\left(\mathfrak{T}, \mathcal{M}^{q}\right)$-pseudo-gate tree of the sentence

$$
\alpha=\mathcal{K} \mathbf{a}_{\mathbf{t}} \neg(\mathbf{q} \wedge \neg \mathbf{q})=\mathcal{K} \mathbf{a}_{\mathbf{t}} \neg(\mathbf{T}(\mathbf{q}, \neg \mathbf{q}, \mathbf{f}))
$$

can be identified with the following sequence consisting of four pseudo-gates:

$$
\left({ }^{\mathfrak{D}} \mathrm{I}^{(1)} \otimes{ }^{\mathfrak{D}} \mathrm{NOT}_{\mathfrak{T}}^{(1)} \otimes{ }^{\mathfrak{D}} \mathrm{I}^{(1)}, \quad{ }^{\mathfrak{D}_{T}} \mathrm{~T}_{\mathfrak{T}}^{(1,1,1)},{ }^{\mathfrak{D}} \mathrm{NOT}_{\mathfrak{T}}^{(3)}, \mathbf{K}_{\mathfrak{a} t}^{(3)}\right) .
$$

Notice that the truth-perspectives $\mathfrak{T}$ and $\mathfrak{T}_{\mathfrak{a}_{\mathfrak{t}}}$ may be different.

The general definition of $\left(\mathfrak{T}, \mathcal{M}^{q}\right)$-pseudo-gate tree is the following:

Definition 2.3. ((T, $\left.\mathcal{M}^{q}\right)$-pseudo-gate tree)

Let $\alpha$ be a sentence such that Height $(\alpha)=k$. The $\left(\mathfrak{T}, \mathcal{M}^{q}\right)$-pseudo-gate tree of $\alpha$ is the sequence of peudo-gates

$$
\operatorname{PsTree} e_{\mathfrak{T}}^{\alpha}=\left({ }^{\alpha} \mathbf{O}_{\mathfrak{T}}^{(k-1)}, \ldots,{ }^{\alpha} \mathbf{O}_{\mathfrak{T}}^{(1)}\right),
$$

that is defined as follows. Suppose that

$$
\text { Level }_{i-1}(\alpha)=\left(\beta_{1}^{\left(r_{1}\right)}, \ldots, \beta_{m}^{\left(r_{m}\right)}\right),
$$

(where $1<i \leq k)$. We put:

$$
{ }^{\alpha} \mathbf{O}_{\mathfrak{T}}^{(i-1)}={ }^{\alpha} \mathbf{X}_{\mathfrak{T}}^{\left(r_{1}\right)} \otimes \ldots \otimes{ }^{\alpha} \mathbf{X}_{\mathfrak{T}}^{\left(r_{m}\right)},
$$


where any ${ }^{\alpha} \mathbf{X}_{\mathfrak{T}}^{\left(r_{j}\right)}$ is a pseudo-gate defined on $\mathcal{H}^{\left(r_{j}\right)}$ such that:

$$
\alpha \mathbf{X}_{\mathfrak{T}}^{\left(r_{j}\right)}=\left\{\begin{array}{l}
\mathfrak{D}^{\left(r_{j}\right)}, \text { if } \beta_{j}^{\left(r_{j}\right)} \text { is atomic; } \\
\mathfrak{D}_{\mathrm{NOT}_{\mathfrak{T}}}^{\left(r_{j}\right)}, \text { if } \beta_{j}^{\left(r_{j}\right)}=\neg \delta ; \\
\mathfrak{D} \sqrt{\mathrm{I}}_{\mathfrak{T}}^{\left(r_{j}\right)}, \text { if } \beta_{j}^{\left(r_{j}\right)}=\sqrt{i d} \delta ; \\
\mathfrak{D} \mathrm{T}_{\mathfrak{T}}^{(u, v, w)}, \text { if } \beta_{j}^{\left(r_{j}\right)}=\mathrm{T}\left(\gamma^{(u)}, \delta^{(v)}, \theta^{(w)}\right) ; \\
\mathfrak{D} \mathbf{X O R}_{\mathfrak{T}}^{(u, v)}, \text { if } \beta_{j}^{\left(r_{j}\right)}=\gamma^{(u)} \biguplus \delta^{(v)} ; \\
\mathbf{U}_{\mathfrak{a}_{\mathfrak{t}}}^{\left(r_{j}\right)}, \text { if } \beta_{j}^{\left(r_{j}\right)}=\mathcal{U} \mathbf{a}_{t} \delta ; \\
\mathbf{K}_{\mathfrak{a}_{\mathfrak{t}}}^{\left(r_{j}\right)}, \text { if } \beta_{j}^{\left(r_{j}\right)}=\mathcal{K} \mathbf{a}_{t} \delta .
\end{array}\right.
$$

Consider now a sentence $\alpha$ and let $\left({ }^{\alpha} \mathbf{O}_{\mathfrak{T}}^{(k-1)}, \ldots,{ }^{\alpha} \mathbf{O}_{\mathfrak{T}}^{(1)}\right)$ be the $\left(\mathfrak{T}, \mathcal{M}^{q}\right)$ pseudo-gate tree of $\alpha$. Any choice of a qumix $\rho$ in $\mathcal{H}^{\alpha}$ determines a sequence $\left(\rho_{k}, \ldots, \rho_{1}\right)$ of qumixes of $\mathcal{H}^{\alpha}$, where:

$$
\begin{gathered}
\rho_{k}=\rho \\
\rho_{k-1}={ }^{\alpha} \mathbf{O}_{\mathfrak{T}}^{k-1}\left(\rho_{k}\right) \\
\vdots \\
\rho_{1}={ }^{\alpha} \mathbf{O}_{\mathfrak{T}}^{1}\left(\rho_{2}\right) .
\end{gathered}
$$

The qumix $\rho_{k}$ can be regarded as a possible input-information concerning the atomic parts of $\alpha$, while $\rho_{1}$ represents the output-information about $\alpha$, given the input-information $\rho_{k}$. Each $\rho_{i}$ corresponds to the information about $\operatorname{Level}_{i}(\alpha)$, given the input-information $\rho_{k}$.

How to determine an information about the parts of $\alpha$ under a given input? It is natural to apply the reduced state function that determines for any state $\rho$ of a composite system $S=S_{1}+\ldots+S_{n}$ the state $\operatorname{Red}^{i_{1}, \ldots, i_{m}}(\rho)$ of any subsystem $S_{i_{1}}+\ldots+S_{i_{m}}$ (where $1 \leq i_{1} \leq n, \ldots, 1 \leq i_{m} \leq n$.) Consider the syntactical tree of $\alpha$ and suppose that:

$$
\text { Level }_{i}(\alpha)=\left(\beta_{i_{1}}, \ldots, \beta_{i_{r}}\right) \text {. }
$$

We know that the $\left(\mathfrak{T}, \mathcal{M}^{q}\right)$-pseudo-gate tree of $\alpha$ and the choice of an input $\rho_{k}\left(\right.$ in $\left.\mathcal{H}^{\alpha}\right)$ determine a sequence of qumixes:

$$
\begin{gathered}
\rho_{k} \leadsto \operatorname{Level}_{k}(\alpha)=\left(\mathbf{q}_{1}, \ldots, \mathbf{q}_{t}\right) \\
\vdots \\
\rho_{i} \leadsto \operatorname{Level}_{i}(\alpha)=\left(\beta_{i_{1}}, \ldots, \beta_{i_{r}}\right) \\
\vdots \\
\rho_{1} \leadsto \operatorname{Level}_{1}(\alpha)=\alpha
\end{gathered}
$$

We can consider $\operatorname{Red}^{j}\left(\rho_{i}\right)$, the reduced information of $\rho_{i}$ with respect to the $j$-th part. From a semantic point of view, this object can be regarded as a contextual information about $\beta_{i_{j}}$ (the subformula of $\alpha$ occurring at the $j$-th position at Level $\left._{i}(\alpha)\right)$ under the input $\rho_{k}$. 
We can now define the notion of holistic model, which assigns meanings to all sentences of the language, for any choice of a truth-perspective $\mathfrak{T}$.

Definition 2.4. (Holistic model)

$A$ holistic model of the language $\mathcal{L}^{E p Q C}$ is a system

$$
\mathcal{M}=\left(T, A g, \text { EpSit, den, }{ }^{\left.\mathcal{M}_{\mathrm{Hol}}\right)}\right.
$$

where:

(1) (T, Ag, EpSit, den) is a quasi-model $\mathcal{M}^{q}$ of the language.

(2) $\mathcal{M}_{\mathrm{Hol}}$ is a map that associates to any truth-perspective $\mathfrak{T}$ a map $\mathcal{M}_{\mathrm{Hol}} \mathrm{I}_{\mathfrak{T}}$ representing a holistic interpretation of the sentences of the language. The following conditions are required.

(2.1) For any sentence $\alpha$, the interpretation ${ }^{\mathcal{M}_{\mathrm{Ho}}} \mathrm{I}_{\mathfrak{T}}$ associates to each level of the syntactical tree of $\alpha$ a meaning, represented by a qumix living in $\mathcal{H}^{\alpha}$ (the semantic space of $\alpha$ );

(2.2) Let $\left({ }^{\alpha} \mathbf{O}_{\mathfrak{T}}^{(k-1)}, \ldots,{ }^{\alpha} \mathbf{O}_{\mathfrak{T}}^{(1)}\right)$ be the $\left(\mathfrak{T}, \mathcal{M}^{q}\right)$-pseudo-gate tree of $\alpha$ and let $1 \leq i<\operatorname{Height}(\alpha)$. Then,

$\mathcal{M}_{\mathrm{Hol}_{\mathfrak{T}}}\left(\right.$ Level $\left._{i}(\alpha)\right)={ }^{\alpha} \mathbf{O}_{\mathfrak{T}}^{(i)}\left({ }^{\mathcal{M}} \operatorname{Hol}_{\mathfrak{T}}\left(\right.\right.$ Level $\left.\left._{i+1}(\alpha)\right)\right)$.

In other words the global meaning of each level (different from the top level) is obtained by applying the corresponding pseudogate to the meaning of the level that occurs immediately above.

(2.3) Let Level $(\alpha)=\left(\beta_{1}, \ldots, \beta_{r}\right)$. Then:

$\beta_{j}=\mathbf{f} \Rightarrow \operatorname{Red}^{j}\left({ }^{\mathcal{M}} \mathrm{Hol}_{\mathfrak{T}}\left(\right.\right.$ Level $\left.\left._{i}(\alpha)\right)\right)={ }^{\mathfrak{T}} P_{0}^{(1)} ;$

$\beta_{j}=\mathbf{t} \Rightarrow \operatorname{Red}^{j}\left(\mathcal{M}_{\mathrm{Hol}_{\mathfrak{T}}}\left(\right.\right.$ Level $\left.\left._{i}(\alpha)\right)\right)={ }^{\mathfrak{T}} P_{1}^{(1)}$, for any $j(1 \leq$ $j \leq r)$.

In other words, the contextual meanings of $\mathbf{f}$ and of $\mathbf{t}$ are always the $\mathfrak{T}$-Falsity and the $\mathfrak{T}$-Truth, respectively.

On this basis, we put:

$$
\mathcal{M}_{\mathrm{Hol}}(\alpha):={ }^{\mathcal{M}_{\mathfrak{T}}} \operatorname{Hol}_{\mathfrak{T}}\left(\text { Level }_{1}(\alpha)\right),
$$

for any sentence $\alpha$.

As an example, consider again the sentence

$$
\alpha=\mathcal{K} \mathbf{a}_{\mathbf{t}} \neg(\mathbf{q} \wedge \neg \mathbf{q})=\mathcal{K} \mathbf{a}_{\mathbf{t}} \neg(\mathbf{T}(\mathbf{q}, \neg \mathbf{q}, \mathbf{f})) .
$$

As we have seen, any $\left(\mathfrak{T}, \mathcal{M}^{q}\right)$-pseudo-gate-tree of $\alpha$ will have the following form:

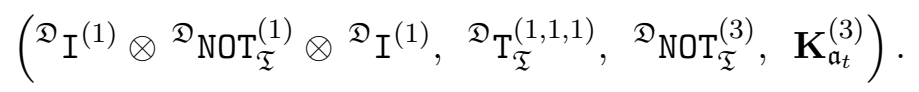

Take a model

$$
\mathcal{M}=\left(T, A g, \text { EpSit, den, }{ }^{\left.\mathcal{M}_{\mathrm{Hol}}\right)}\right.
$$

such that:

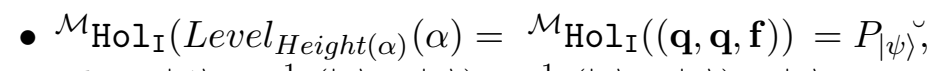
where $|\psi\rangle=\frac{1}{\sqrt{2}}(|0\rangle+|1\rangle) \otimes \frac{1}{\sqrt{2}}(|0\rangle+|1\rangle) \otimes|0\rangle$. 
- EpSit assigns to agent $\mathfrak{a}_{\mathfrak{t}}$ the epistemic situation

$$
\left(\mathfrak{T}_{\mathfrak{a}_{\mathfrak{t}}}, \operatorname{Ep} D_{\mathfrak{a}_{\mathfrak{t}}}, \mathbf{U}_{\mathfrak{a}_{t}}, \mathbf{K}_{\mathfrak{a}_{t}}\right),
$$

where $E p D_{\mathfrak{a}_{\mathfrak{t}}}=\mathfrak{D}$ and $\mathbf{K}_{\mathfrak{a}_{t}}(\rho)=\rho$, for any $\rho \in \mathfrak{D}$. In other words, $\mathfrak{a}_{\mathfrak{t}}$ has a maximal epistemic capacity ${ }^{3}$.

We obtain:

$\mathcal{M}_{\mathrm{Hol}_{\mathrm{I}}}\left(\mathcal{K} \mathbf{a}_{t} \neg(\mathbf{q} \wedge \neg \mathbf{q})\right)=P_{|\varphi\rangle}$, where:

$|\varphi\rangle=\mathbf{K}_{\mathfrak{a}_{\mathfrak{t}}}^{(3)} \mathrm{NOT}_{\mathrm{I}}^{(3)} \mathrm{T}_{\mathrm{I}}^{(1,1,1)}\left(\mathrm{I}^{(1)} \otimes \operatorname{NOT}_{\mathrm{I}}^{(1)} \otimes \mathrm{I}^{(1)}\right)\left(\frac{1}{\sqrt{2}}(|0\rangle+|1\rangle) \otimes \frac{1}{\sqrt{2}}(|0\rangle+|1\rangle) \otimes\right.$

$|0\rangle)=\frac{1}{2}(|0,1,1\rangle+|0,0,1\rangle+|1,1,0\rangle+|1,0,1\rangle)$.

Hence, $\mathrm{p}_{\mathrm{I}}\left(\mathcal{M}^{\mathrm{Hol}} \mathrm{I}_{\mathrm{I}}\left(\mathcal{K} \mathbf{a}_{t} \neg(\mathbf{q} \wedge \neg \mathbf{q})\right)\right)=\frac{3}{4} \neq 1$.

This example clearly shows how even an agent with a maximal epistemic capacity does not necessarily know a very simple instance of the non-contradiction principle!

Unlike standard compositional semantics, any $\mathcal{M}_{\mathrm{Hol}}(\alpha)$ represents a kind of autonomous semantic context that is not necessarily correlated with the meanings of other sentences. At the same time, given a sentence $\gamma$, $\mathcal{M}_{\mathrm{Hol}} \mathrm{I}_{\mathfrak{T}}$ determines the contextual meaning, with respect to the context $\mathcal{M}_{\mathrm{Hol}}(\gamma)$, of any occurrence of a subformula $\beta$ in the syntactical tree of $\gamma$.

Definition 2.5. (Contextual meaning)

Consider a sentence $\gamma$ such that

$$
\text { Level }_{i}(\gamma)=\left(\beta_{i_{1}}, \ldots, \beta_{i_{r}}\right) .
$$

The contextual meaning of the occurrence $\beta_{i_{j}}$ with respect to the context $\mathcal{M}_{\mathrm{Hol}}(\gamma)$ is defined as follows:

$$
\mathcal{M}_{\mathrm{Hol}}^{\gamma}\left(\beta_{i_{j}}\right):=\operatorname{Red}^{j}\left({ }^{\mathcal{M}} \operatorname{Hol}_{\mathfrak{T}}\left(\text { Level }_{i}(\gamma)\right)\right) .
$$

Hence, in particular, we have for any sentence $\gamma$

$$
\mathcal{M}_{\mathrm{Hol}} \gamma(\gamma)=\mathcal{M}_{\mathfrak{T}} \mathrm{Hol}_{\mathfrak{T}}\left(\text { Level }_{1}(\gamma)\right)=\mathcal{M}_{\mathrm{Hol}}(\gamma) .
$$

Generally, different occurrences $\beta_{i_{j}}$ and $\beta_{h_{k}}$ of one and the same subformula $\beta$ in the syntactical tree of $\gamma$ may receive different contextual meanings. In other words, we may have:

$$
\mathcal{M}_{\mathrm{Hol}} \underset{\mathfrak{T}}{\gamma}\left(\beta_{i_{j}}\right) \neq \mathcal{M}_{\mathrm{Hol}_{\mathfrak{T}}}^{\gamma}\left(\beta_{h_{k}}\right) .
$$

When this is not the case, we will say that one is dealing with a normal model.

Definition 2.6. (Normal holistic model)

A normal holistic model of the language $\mathcal{L}^{E p Q C}$ is a holistic model $\mathcal{M}$ such that for any truth-perspective $\mathfrak{T}$ and for any sentence $\gamma$, the interpretation $\mathcal{M}_{\mathrm{Ho}} \mathrm{I}_{\mathfrak{T}}$ determines for any occurrence $\beta_{i_{j}}$ of a subformula $\beta$ of $\gamma$ in the syntactical tree of $\gamma$ the same contextual meaning, which will be uniformly indicated by ${ }^{\mathcal{M}_{\mathrm{Hol}}} \underset{\mathfrak{T}}{\gamma}(\beta)$.

\footnotetext{
${ }^{3}$ See Section 3 of the first Part of this article.
} 
In the following we will always refer to normal holistic models.

Suppose that $\beta$ is a subformula of two different formulas $\gamma$ and $\delta$. Generally, we have:

$$
\mathcal{M}_{\mathrm{Hol}}^{\gamma}(\beta) \neq \mathcal{M}_{\mathfrak{T}} \mathrm{Hol}_{\mathfrak{T}}^{\delta}(\beta) .
$$

In other words, sentences may receive different contextual meanings in different contexts also in the case of the normal holistic semantics.

To what extent do contextual meanings and gates (associated to the logical connectives) commute? An answer to this question is given by the following theorem.

Theorem 2.1. Consider a holistic model $\mathcal{M}=\left(T, A g\right.$, EpSit, den, ${ }^{\mathcal{M}}$ Hol $)$ and a truth-perspective $\mathfrak{T}$.

1. Let $\neg \alpha$ be a subformula of $\gamma$. Suppose that $\neg \alpha=\beta_{i_{j}}$ (the formula occurring at the $j$-th position of the $i$-th level in the syntactical tree of $\gamma)$, while $\alpha=\beta_{(i+1)_{k}}$. We have:

$$
\begin{aligned}
& \mathcal{M}_{\mathrm{Hol}_{\mathfrak{T}}^{\gamma}}^{\gamma}(\neg \alpha)=\operatorname{Red}\left({ }^{j} \mathcal{M}_{\mathrm{Hol}_{\mathfrak{T}}}\left(\text { Level }_{i}(\gamma)\right)\right)= \\
& \mathfrak{D}_{\operatorname{NOT}_{\mathfrak{T}}}^{(\operatorname{Att}(\alpha))}\left(\operatorname{Red}^{k}\left(\mathcal{M}_{\mathrm{Hol}}\left(\text { Level }_{i+1}(\gamma)\right)\right)\right)= \\
& \left.\mathfrak{D}_{\left.\operatorname{NOT}_{\mathfrak{T}}^{(A t}(\alpha)\right)}^{\left(\mathcal{M}_{\mathrm{Hol}}\right.} \mathbf{1}_{\mathfrak{T}}^{\gamma}(\alpha)\right) .
\end{aligned}
$$

2. Let $\sqrt{i d} \alpha$ be a subformula of $\gamma$. Suppose that $\sqrt{i d} \alpha=\beta_{i_{j}}$, while $\alpha=\beta_{(i+1)_{k}}$. We have:

$$
\begin{aligned}
& \mathcal{M}_{\mathrm{Hol}_{\mathfrak{T}}}^{\gamma}(\sqrt{i d} \alpha)=\operatorname{Red}^{j}\left(\mathcal{M}_{\operatorname{Hol}_{\mathfrak{T}}}\left(\operatorname{Level}_{i}(\gamma)\right)\right)= \\
& \mathfrak{D}{\sqrt{I_{\mathfrak{T}}}}^{(\operatorname{At}(\alpha))}\left(\operatorname{Red}^{k}\left(\mathcal{M}_{\mathrm{Hol}}\left(\text { Level }_{i+1}(\gamma)\right)\right)\right)= \\
& \mathfrak{D} \sqrt{\mathrm{I}}_{\mathfrak{T}}^{(A t(\alpha))}\left(\mathcal{M}_{\mathrm{Hol}} \mathbf{l}_{\mathfrak{T}}^{\gamma}(\alpha)\right) \text {. }
\end{aligned}
$$

3. Let $\mathrm{T}\left(\alpha_{1}, \alpha_{2}, \alpha_{3}\right)$ be a subformula of $\gamma$. Suppose that in the syntactical tree of $\gamma: \mathrm{T}\left(\alpha_{1}, \alpha_{2}, \alpha_{3}\right)=\beta_{i_{j}}$, while $\alpha_{1}=\beta_{(i+1)_{k_{1}}}, \alpha_{2}=\beta_{(i+1)_{k_{2}}}$, $\alpha_{3}=\beta_{(i+1)_{k_{3}}}$ We have:

$\mathcal{M}_{\mathrm{Hol}} \mathbf{T}_{\mathfrak{T}}^{\gamma}\left(\mathbf{T}\left(\alpha_{1}, \alpha_{2}, \alpha_{3}\right)\right)=\operatorname{Red}^{j}\left(\mathcal{M}_{\operatorname{Hol}_{\mathfrak{T}}}\left(\operatorname{Level}_{i}(\gamma)\right)\right)=$ $\mathfrak{D}_{\mathrm{T}_{\mathfrak{T}}}^{\left(A t\left(\alpha_{1}\right), \operatorname{At}\left(\alpha_{2}\right), \operatorname{At}\left(\alpha_{3}\right)\right)}\left(\operatorname{Red}^{k_{1}, k_{2}, k_{3}}\left(\mathcal{M}_{\mathrm{Hol}_{\mathfrak{T}}}\left(\right.\right.\right.$ Level $\left.\left.\left._{i+1}(\gamma)\right)\right)\right)$.

4. Let $\alpha_{1} \uplus \alpha_{2}$ be a subformula of $\gamma$. Suppose that in the syntactical tree of $\left.\gamma: \alpha_{1} \uplus \alpha_{2}\right)=\beta_{i_{j}}$, while $\alpha_{1}=\beta_{(i+1)_{k_{1}}}, \alpha_{2}=\beta_{(i+1)_{k_{2}}}$. We have:

$\mathcal{M}_{\mathrm{Hol}_{\mathfrak{T}}}^{\gamma}\left(\alpha_{1} \uplus \alpha_{2}\right)=\operatorname{Red}^{j}\left(\mathcal{M}_{\mathrm{Hol}_{\mathfrak{T}}}\left(\operatorname{Level}_{i}(\gamma)\right)\right)=$ ${ }^{\mathfrak{D}} \operatorname{XOR}_{\mathfrak{T}}^{\left(\operatorname{At}\left(\alpha_{1}\right), \operatorname{At}\left(\alpha_{2}\right)\right)}\left(\operatorname{Red}^{k_{1}, k_{2}}\left(\mathcal{M}^{\mathrm{Hol}_{\mathfrak{T}}}\left(\right.\right.\right.$ Level $\left.\left.\left._{i+1}(\gamma)\right)\right)\right)$.

5. Let $\mathcal{U}_{\mathbf{a}_{t}} \alpha$ be a subformula of $\gamma$. Suppose that $\mathcal{U}_{\mathbf{a}_{t}} \alpha=\beta_{i_{j}}$, while $\alpha=\beta_{(i+1)_{k}}$. We have:

$$
\begin{aligned}
& \mathcal{M}_{\mathrm{Hol}_{\mathfrak{T}}}^{\gamma}\left(\mathcal{U}_{\mathbf{a}_{t}} \alpha\right)=\operatorname{Red}^{j}\left(\mathcal{M}_{\operatorname{Hol}_{\mathfrak{T}}}\left(\operatorname{Level}_{i}(\gamma)\right)\right)= \\
& \mathbf{U}_{\mathfrak{a}_{\mathfrak{t}}}^{(\operatorname{At}(\alpha))}\left(\operatorname{Red}^{k}\left(\mathcal{M}_{\mathrm{Hol}_{\mathfrak{T}}}\left(\operatorname{Level}_{i+1}(\gamma)\right)\right)=\mathbf{U}_{\mathfrak{a}_{\mathfrak{t}}}^{(A t(\alpha))}\left(\mathcal{M}_{\mathrm{Hol}_{\mathfrak{T}}}^{\gamma}(\alpha)\right) .\right.
\end{aligned}
$$

6. Let $\mathcal{K}_{\mathbf{a}_{t}} \alpha$ be a subformula of $\gamma$. Suppose that $\mathcal{K}_{\mathbf{a}_{t}} \alpha=\beta_{i_{j}}$, while $\alpha=\beta_{(i+1)_{k}}$. We have:

$$
\begin{aligned}
& \mathcal{M}_{\operatorname{Hol}_{\mathfrak{T}}}^{\gamma}\left(\mathcal{K}_{\mathbf{a}_{t}} \alpha\right)=\operatorname{Red}^{j}\left(\mathcal{M}_{\mathrm{Hol}_{\mathfrak{T}}}\left(\operatorname{Level}_{i}(\gamma)\right)\right)= \\
& \mathbf{K}_{\mathfrak{a}_{\mathfrak{t}}}^{(\operatorname{At}(\alpha))}\left(\operatorname{Red}^{k}\left(\mathcal{M}_{\operatorname{Hol}_{\mathfrak{T}}}\left(\operatorname{Level}_{i+1}(\gamma)\right)\right)=\mathbf{K}_{\mathfrak{a}_{\mathfrak{t}}}^{(\operatorname{At}(\alpha))}\left(\mathcal{M}_{\operatorname{Hol}_{\mathfrak{T}}}^{\gamma}(\alpha)\right) .\right.
\end{aligned}
$$


Proof. By definition of syntactical tree, of pseudo-gate tree, of normal holistic model and of contextual meaning.

Notice that, generally, the contextual meaning of a conjunction is not the conjunction of the contextual meanings of the two members. As a counterexample, consider the following contradictory sentence:

$$
\gamma=\mathbf{q} \wedge \neg \mathbf{q}=\mathbf{T}(\mathbf{q}, \neg \mathbf{q}, \mathbf{f}),
$$

whose syntactical tree is:

$$
\begin{gathered}
\operatorname{Level}_{3}(\gamma)=(\mathbf{q}, \mathbf{q}, \mathbf{f}) \\
\operatorname{Level}_{2}(\gamma)=(\mathbf{q}, \neg \mathbf{q}, \mathbf{f}) \\
\operatorname{Level}_{1}(\gamma)=\mathrm{T}(\mathbf{q}, \neg \mathbf{q}, \mathbf{f})
\end{gathered}
$$

Consider a model $\mathcal{M}$ such that:

$$
\mathcal{M}_{\mathrm{Hol}_{\mathrm{I}}}\left(\text { Level }_{3}(\gamma)\right)=P_{\frac{1}{\sqrt{2}}(|0,1,0\rangle+|1,0,0\rangle)}
$$

(which is a maximally entangled quregister with respect to the first and to the second part 4 ). Hence:

$$
\mathcal{M}_{\mathrm{Hol}_{\mathrm{I}}}\left(\operatorname{Level}_{1}(\gamma)\right)=\mathcal{M}_{\mathrm{Hol}_{\mathrm{I}}}(\gamma)=\mathcal{M}_{\mathrm{Hol}}^{\gamma}(\gamma)=P_{\frac{1}{\sqrt{2}}(|0,0,0\rangle+|1,1,1\rangle)}
$$

(which is a maximally entangled quregister). At the same time, we have:

$$
\mathcal{M}_{\mathrm{Hol}}^{\gamma}(\mathbf{q})=\mathcal{M}_{\mathrm{Hol}}^{\gamma}(\neg \mathbf{q})=\frac{1}{2} P_{0}^{(1)}+\frac{1}{2} P_{1}^{(1)},
$$

which is a proper mixture. Consequently:

$$
\mathfrak{D}_{\mathrm{T}}^{(1,1,1)}\left(\mathcal{M}_{\mathrm{Hol}}^{\gamma}(\mathbf{q}) \otimes \mathcal{M} \mathrm{Hol}_{\mathrm{I}}^{\gamma}(\neg \mathbf{q}) \otimes \mathcal{M} \mathrm{Hol}_{\mathrm{I}}^{\gamma}(\mathbf{f})\right) \neq \mathcal{M}_{\mathrm{Hol}} \mathrm{I}_{\mathrm{I}}^{\gamma}(\mathbf{T}(\mathbf{q}, \neg \mathbf{q}, \mathbf{f})) \text {. }
$$

Notice that: $\mathrm{p}_{\mathrm{I}}\left(\mathcal{M}^{\mathrm{Hol}_{\mathrm{I}}}(\mathbf{q} \wedge \neg \mathbf{q})\right)=\frac{1}{2}$; while: $\mathrm{p}_{\mathrm{I}}\left({ }^{\mathfrak{D}} \mathrm{T}^{(1,1,1,)}\left(\mathcal{M}_{\mathrm{Hol}}^{\gamma}(\mathbf{q}) \otimes \mathcal{M}^{\mathrm{Hol}} \mathrm{I}_{\mathrm{I}}^{\gamma}(\neg \mathbf{q}) \otimes \mathcal{M}_{\mathrm{Hol}}^{\gamma}(\mathbf{f})\right)\right)=\frac{1}{4}$.

Definition 2.7. (Harmonic and sound models) Let $\mathcal{M}=\left(T, A g\right.$, EpSit, den, ${ }^{\mathcal{M}}$ Hol $)$ be a model.

- $\mathcal{M}$ is called harmonic iff the epistemic structure of $\mathcal{M}$ is harmonic, i.e. all agents of the structure share the same truth-perspective $\mathfrak{T}$. Furthermore, the interpretation-function ${ }^{\mathcal{M}} \mathrm{Hol}$ is only defined for the truth-perspective $\mathfrak{T}$.

- $\mathcal{M}$ is called sound iff all agents $\mathfrak{a}_{\mathfrak{t}}$ of $\mathcal{M}$ have a sound epistemic capacity (i.e. assign the "right" probability-values to the truth-values of their truth-perspectives) 5

By harmonic epistemic quantum computational semantics (sound epistemic quantum computational semantics) we will mean the semantics that only refers to harmonic models (sound models).

We can now define the notions of truth, validity and logical consequence.

\footnotetext{
${ }^{4}$ See Section 2 of the first Part of this article.

${ }^{5}$ See Section 3 of the first Part of this article.
} 
Definition 2.8. (Contextual truth)

Let $\alpha$ be a subformula of $\gamma$.

$\vDash_{(\gamma, \mathcal{M}, \mathfrak{T})} \alpha$ (the sentence $\alpha$ is true with respect to the context $\gamma$, the model $\mathcal{M}$ and the truth-perspective $\mathfrak{T})$ iff $\mathrm{p}_{\mathfrak{T}}\left(\mathcal{M}_{\mathrm{Ho}} \mathbf{H}_{\mathfrak{T}}^{\gamma}(\alpha)\right)=1$.

Definition 2.9. (Truth)

$\vDash_{(\mathcal{M}, \mathfrak{T})} \alpha$ (the sentence $\alpha$ is true with respect to the model $\mathcal{M}$ and the truthperspective $\mathfrak{T})$ iff $\vDash_{(\alpha, \mathcal{M}, \mathfrak{T})} \alpha$.

Hence, the concept of truth turns out to be a special case of the concept of contextual truth.

Definition 2.10. (Contextual validity)

Let $\alpha$ be a subformula of $\gamma$.

- $\vDash_{(\gamma, \mathfrak{T})} \alpha$ (the sentence $\alpha$ is valid with respect to the context $\gamma$ and the truth-perspective $\mathfrak{T})$ iff for any model $\mathcal{M}, \vDash_{(\gamma, \mathcal{M}, \mathfrak{T})} \alpha$.

- $\vDash_{\gamma} \alpha$ (the sentence $\alpha$ is valid with respect to the context $\gamma$ ) iff for any truth-perspective $\mathfrak{T}, \vDash_{(\gamma, \mathfrak{T})} \alpha$.

Definition 2.11. (Validity)

- $\vDash_{\mathfrak{T}} \alpha$ (the sentence $\alpha$ is valid with respect to the truth-perspective $\mathfrak{T}$ ) iff $\vDash_{(\alpha, \mathfrak{T})} \alpha$.

- $\vDash \alpha$ (the sentence $\alpha$ is valid) iff $\vDash_{\alpha} \alpha$.

Definition 2.12. (Consequence with respect to a quasi-model $\mathcal{M}^{q}$ )

Let $\gamma$ be a sentence such that $\alpha$ and $\beta$ are subformulas of $\gamma$ and let $\mathfrak{T}$ be a truth-perspective.

- $\alpha \vDash_{\left(\gamma, \mathcal{M}^{q}, \mathfrak{T}\right)} \beta$ (the sentence $\beta$ is a consequence of the sentence $\alpha$ with respect to the context $\gamma$, the quasi-model $\mathcal{M}^{q}$ and the truthperspective $\mathfrak{T}$ ) iff for any model $\mathcal{M}$ based on $\mathcal{M}^{q}$ :

$$
\vDash_{(\gamma, \mathcal{M}, \mathfrak{T})} \alpha \Rightarrow \vDash_{(\gamma, \mathcal{M}, \mathfrak{T})} \beta .
$$

- $\alpha \vDash_{\left(\gamma, \mathcal{M}^{q}\right)} \beta$ (the sentence $\beta$ is a consequence of the sentence $\alpha$ with respect to the context $\gamma$ and the quasi-model $\mathcal{M}^{q}$ ) iff for any truthperspective $\mathfrak{T}, \alpha \vDash_{\left(\gamma, \mathcal{M}^{q}, \mathfrak{T}\right)} \beta$.

Definition 2.13. Logical consequence

Let $\gamma$ be a sentence such that $\alpha$ and $\beta$ are subformulas of $\gamma$ and let $\mathfrak{T}$ be a truth-perspective.

- $\alpha \vDash_{(\gamma, \mathfrak{T})} \beta$ ( $\beta$ is a logical consequence of $\alpha$ with respect to the context $\gamma$ and the truth-perspective $\mathfrak{T})$ iff for any quasi-model $\mathcal{M}^{q}$, $\alpha \vDash_{\left(\gamma, \mathcal{M}^{q}, \mathfrak{T}\right)} \beta$.

- $\alpha \vDash_{\gamma} \beta$ ( $\beta$ is a logical consequence of $\alpha$ with respect to the context $\gamma$ ) iff for any truth-perspective $\mathfrak{T}, \alpha \vDash_{(\gamma, \mathfrak{T})} \beta$.

- $\alpha \vDash \beta$ ( $\beta$ is a logical consequence of $\alpha$ iff for any context $\gamma$ such that $\alpha$ and $\beta$ are subformulas of $\gamma$ and $\alpha \vDash_{\gamma} \beta$. 
The concepts of consequence and of logical consequence, defined above, correspond to weak concepts, being defined in terms of $\mathfrak{T}$-Truth, and not in terms of the preorder relation $\preceq_{\mathfrak{T}}$ (as one could expect). From an intuitive point of view, however, such weak notions turn out to be more interesting in the case of epistemic situations described in the framework of a holistic semantics.

Notice that only the contextual notion of logical consequence turns out to satisfy transitivity $\left(\alpha \vDash_{\gamma} \beta\right.$ and $\beta \vDash_{\gamma} \delta \Rightarrow \alpha \vDash_{\gamma} \delta$ ). Full transitivity $(\alpha \vDash \beta$ and $\beta \vDash \delta \Rightarrow \alpha \vDash \delta)$ is naturally violated in the holistic semantics.

As expected, in the particular case of the harmonic epistemic semantics (where all agents share the same truth-perspective) the definitions of truth, validity and logical consequence can be simplified, since the reference to $\mathfrak{T}$ is no longer necessary. Accordingly, in such a case we will write:

$\vDash_{(\gamma, \mathcal{M})}^{\operatorname{Harm}} \alpha ; \vDash_{\mathcal{M}}^{\text {Harm }} \alpha$ (harmonic truth);

$\vDash_{\gamma}^{\text {Harm }} \alpha ; \vDash^{\text {Harm }} \alpha$ (harmonic validity);

$\alpha \models_{\left(\gamma, \mathcal{M}^{q}\right)}^{\operatorname{Harm}} \beta ; \alpha \models_{\gamma}^{\text {Harm }} \beta ; \alpha \vDash^{\text {Harm }} \beta$ (harmonic logical consequence).

\section{Some EPISTEMic Situations}

We will now illustrate some significant examples of epistemic situations that arise in this semantics. We will always refer to models

$$
\mathcal{M}=\left(T, A g, \mathbf{E p S i t}, \operatorname{den},{ }^{\mathcal{M}} \mathrm{Hol}\right)
$$

such that $\operatorname{den}(\mathbf{a})=\mathfrak{a} ; \operatorname{den}(t)=\mathfrak{t}$.

1) $\mathcal{K} \mathbf{a}_{t} \alpha \vDash^{\text {Harm }} \alpha$.

In the harmonic semantics, sentences that are known by a given agent at a given time are true.

1 ) is an immediate consequence of the definition of logical consequence and of Theorem 2.1.

2) As a particular case of 1) we obtain:

$\mathcal{K} \mathbf{a}_{t} \alpha \mathcal{K} \mathbf{a}_{t} \alpha \vDash^{\operatorname{Harm}} \mathcal{K} \mathbf{a}_{t} \alpha$.

Knowing of knowing implies knowing. But not the other way around!

3) In the non-harmonic semantics only the two following conditions (which are weaker than 1) and 2)) hold for any quasi-model $\mathcal{M}^{q}$ and any agent $\mathfrak{a}_{\mathfrak{t}}$ of $\mathcal{M}^{q}$ :

3.1) $\mathcal{K} \mathbf{a}_{t} \alpha \vDash_{\left(\mathcal{M}^{q}, \mathfrak{T}_{\mathfrak{a}_{\mathfrak{t}}}\right)} \alpha$;

3.2) $\mathcal{K} \mathbf{a}_{t} \alpha \mathcal{K} \mathbf{a}_{t} \alpha \vDash{ }_{\left(\mathcal{M}^{q}, \mathfrak{T}_{\mathfrak{a}}\right)} \mathcal{K} \mathbf{a}_{t} \alpha$.

4) $\mathcal{K} \mathbf{a}_{t} \mathcal{K} \mathbf{b}_{t} \alpha \vDash^{\text {Harm }} \alpha$.

In the harmonic semantics, knowing that another agent knows a given sentence implies that the sentence in question holds. At the same time, we will have:

$\mathcal{K} \mathbf{a}_{t} \mathcal{K} \mathbf{b}_{t} \alpha \not{ }^{H a r m} \mathcal{K} \mathbf{a}_{t} \alpha$.

Alice might know that Bob knows a given sentence, without knowing herself the sentence in question! 
5) In the harmonic sound semantics (where for any agent $\mathfrak{a}_{\mathfrak{t}}, \mathbf{K}_{\mathfrak{a}_{\mathfrak{t}}} \mathfrak{T}_{\mathfrak{a}_{\mathfrak{t}}} P_{1}^{(1)}=$ $\mathfrak{T}_{\mathfrak{a}_{\mathfrak{t}}} P_{1}^{(1)}$ and $\mathbf{K}_{\mathfrak{a}_{\mathfrak{t}}} \mathfrak{T}_{\mathfrak{a}_{\mathfrak{t}}} P_{0}^{(1)}=\mathfrak{T}_{\mathfrak{a}_{\mathfrak{t}}} P_{0}^{(1)}$ ) we have:

$$
\vDash^{\text {Harm }} \mathcal{K} \mathbf{a}_{t} \mathbf{t} ; \vDash^{\text {Harm }} \mathcal{K} \mathbf{a}_{t} \neg \mathbf{f} .
$$

Hence, there are sentences that every agent knows.

6) $\mathcal{K} \mathbf{a}_{t}(\alpha \wedge \beta) \not \models \mathcal{K} \mathbf{a}_{t} \alpha ; \mathcal{K} \mathbf{a}_{t}(\alpha \wedge \beta) \not \models \mathcal{K} \mathbf{a}_{t} \beta$.

Knowing a conjunction does not generally imply knowing its members.

7) $\vDash_{(\gamma, \mathcal{M})} \mathcal{K} \mathbf{a}_{t} \alpha$ and $\vDash_{(\gamma, \mathcal{M})} \mathcal{K} \mathbf{a}_{t} \beta \nRightarrow \vDash_{(\gamma, \mathcal{M})} \mathcal{K} \mathbf{a}_{t}(\alpha \wedge \beta)$.

Knowledge is not generally closed under conjunction.

8) Let $\mathcal{M}$ be a model and let $\mathfrak{a}_{\mathfrak{t}}$ be an agent of $\mathcal{M}$.

$\not F_{\left(\mathcal{M}, \mathfrak{T}_{\mathfrak{a}_{\mathfrak{f}}}\right)} \mathcal{K} \mathbf{a}_{t}(\alpha \wedge \neg \alpha)$.

Contradictions are never known.

In order to prove 8$)$, suppose, by contradiction, that there exists a model $\mathcal{M}$ and an agent $\mathfrak{a}_{\mathfrak{t}}$ such that: $\vDash_{\left(\mathcal{M}, \mathfrak{T}_{\mathfrak{a}_{\mathfrak{t}}}\right)} \mathcal{K} \mathbf{a}_{t}(\alpha \wedge \neg \alpha)$.

Then, $\mathbf{p}_{\mathfrak{T}_{\mathfrak{a}_{t}}}\left(\mathcal{M}_{\mathrm{Hol}_{\mathfrak{T}_{\mathfrak{a}_{t}}}}\left(\mathcal{K}_{\mathbf{a}_{t}}(\alpha \wedge \neg \alpha)\right)\right)=1$.

By definition of model and by Theorem 2.1 we have:

$\mathcal{M}_{\mathrm{Ho}} \mathbf{l}_{\mathfrak{T}_{\mathfrak{a}}}\left(\mathcal{K} \mathbf{a}_{t}(\alpha \wedge \neg \alpha)\right)=\mathbf{K}_{\mathfrak{a}_{\mathfrak{t}}}\left(\mathcal{M}_{\mathrm{Hol}_{\mathfrak{T}_{\mathfrak{t}}}}(\alpha \wedge \neg \alpha)\right)$.

Consequently, by hypothesis, $\mathbf{p}_{\mathfrak{T}_{\mathfrak{a}_{\mathfrak{t}}}}\left(\mathbf{K}_{\mathfrak{a}_{\mathfrak{t}}}\left(\mathcal{M}_{\mathrm{Hol}_{\mathfrak{T}_{\mathfrak{a}_{\mathfrak{t}}}}}(\alpha \wedge \neg \alpha)\right)\right)=1$.

Thus, by the properties of knowledge operations:

$\mathrm{p}_{\mathfrak{T}_{\mathfrak{a}_{\mathfrak{t}}}}\left(\mathcal{M}_{\mathrm{Hol}} \boldsymbol{I}_{\mathfrak{T}_{\mathfrak{t}}}(\alpha \wedge \neg \alpha)\right)=1$, which is impossible, owing to the following Lemma (of the holistic semantics).

Lemma 3.1. For any sentence $\alpha$, for any truth-perspective $\mathfrak{T}$ and for any holistic model $\mathcal{M}$,

$$
\operatorname{p}_{\mathfrak{T}}\left({ }^{\mathcal{M}}{ }_{\mathrm{Hol}}(\alpha \wedge \neg \alpha)\right) \neq 1 .
$$

9) In the non-harmonic semantics the following situation is possible:

$\vDash_{\left(\mathcal{M}, \mathfrak{T}_{\mathfrak{a}}\right)} \mathcal{K} \mathbf{a}_{t} \mathcal{K} \mathbf{b}_{t} \mathbf{f}$.

In other words, according to the truth-perspective of Alice it is true that Alice (at time $t$ ) knows that Bob (at time $t$ ) knows the Falsity of Alice's truth-perspective.

As an example, consider a (non-harmonic) model $\mathcal{M}$ with two agents $\mathfrak{a}_{\mathfrak{t}}$ and $\mathfrak{b}_{\mathfrak{t}}$ satisfying the following conditions:

a) the epistemic distance between the truth-perspectives of $\mathfrak{a}_{\mathfrak{t}}$ and of $\mathfrak{b}_{\mathfrak{t}}$ is greater than or equal to $\frac{1}{2} 6$ In such a case we have: $\mathfrak{T}_{\mathfrak{a}_{\mathfrak{t}}} P_{1}^{(1)} \preceq_{\mathfrak{T}_{\mathfrak{b}_{\mathfrak{t}}}} \mathfrak{T}_{\mathfrak{a}_{\mathfrak{t}}} P_{0}^{(1)}$

(according to Bob's truth-perspective, Alice's Truth precedes Alice's Falsity);

b) $\mathbf{K}_{\mathfrak{b}_{\mathfrak{t}}} \mathfrak{T}_{\mathfrak{a}_{\mathfrak{t}}} P_{0}^{(1)}=\mathfrak{T}_{\mathfrak{a}_{\mathfrak{t}}} P_{1}^{(1)}$

(the information according to which Bob knows Alice's Falsity is true with respect to Alice's truth-perspective);

\footnotetext{
${ }^{6}$ The concept of epistemic distance has been defined in Section 2 of the first Part of this article.
} 
c) $\mathbf{K}_{\mathfrak{a}_{\mathfrak{t}}} \mathfrak{T}_{\mathfrak{a}_{\mathfrak{t}}} P_{1}^{(1)}=\mathfrak{T}_{\mathfrak{a}_{\mathfrak{t}}} P_{1}^{(1)}$

(Alice at time $\mathfrak{t}$ has a sound epistemic capacity).

Consider the syntactical tree of $\mathcal{K} \mathbf{a}_{t} \mathcal{K} \mathbf{b}_{t} \mathbf{f}$ :

$$
\begin{gathered}
\text { Level }_{3}\left(\mathcal{K} \mathbf{a}_{t} \mathcal{K} \mathbf{b}_{t} \mathbf{f}\right)=\mathbf{f} \\
\text { Level }_{2}\left(\mathcal{K} \mathbf{a}_{t} \mathcal{K} \mathbf{b}_{t} \mathbf{f}\right)=K \mathbf{b}_{t} \mathbf{f} \\
\text { Level }_{1}\left(\mathcal{K} \mathbf{a}_{t} \mathcal{K} \mathbf{b}_{t} \mathbf{f}\right)=\mathcal{K} \mathbf{a}_{t} \mathcal{K} \mathbf{b}_{t} \mathbf{f}
\end{gathered}
$$

The qumixes assigned by ${ }^{\mathcal{M}} \mathrm{Hol}_{\mathfrak{T}_{\mathfrak{a}_{\mathfrak{t}}}}$ to the levels of this tree are:

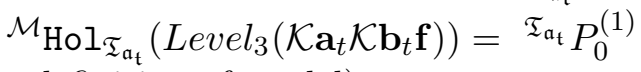

(by definition of model);

$\mathcal{M}_{\mathrm{Hol}_{\mathfrak{T}_{\mathfrak{a}_{\mathfrak{t}}}}}$ Level $\left._{2}\left(\mathcal{K} \mathbf{a}_{t} \mathcal{K} \mathbf{b}_{t} \mathbf{f}\right)\right)=\mathbf{K}_{\mathfrak{b}_{\mathfrak{t}}}\left(\mathcal{M}_{\mathrm{Hol}_{\mathfrak{T}_{\mathfrak{t}}}}\left(\right.\right.$ Level $\left._{3}\left(\mathcal{K} \mathbf{a}_{t} \mathcal{K} \mathbf{b}_{t} \mathbf{f}\right)\right)=\mathfrak{T}_{\mathfrak{a}_{\mathfrak{t}}} P_{1}^{(1)}$ (by definition of model and by $\mathrm{b})$ );

$\mathcal{M}_{\mathrm{Hol}_{\mathfrak{T}_{\mathfrak{t}}}}\left(\right.$ Level $\left._{1}\left(\mathcal{K} \mathbf{a}_{t} \mathcal{K} \mathbf{b}_{t} \mathbf{f}\right)\right)=\mathbf{K}_{\mathfrak{b}_{\mathfrak{t}}}\left(\mathcal{M}_{\mathrm{Hol}_{\mathfrak{T}_{\mathfrak{a}_{\mathfrak{t}}}}}\left(\right.\right.$ Level $\left._{2}\left(\mathcal{K} \mathbf{a}_{t} \mathcal{K} \mathbf{b}_{t} \mathbf{f}\right)\right)=\mathfrak{T}_{\mathfrak{a}_{\mathfrak{t}}} P_{1}^{(1)}$ (by definition of model and by c)).

Hence, $\mathrm{p}_{\mathfrak{T}_{\mathfrak{a}_{\mathfrak{t}}}}\left(\mathcal{M}_{\mathrm{Hol}} \mathbf{I}_{\mathfrak{T}_{\mathfrak{a}_{\mathfrak{t}}}}\left(\mathcal{K} \mathbf{a}_{t} \mathcal{K} \mathbf{b}_{t} \mathbf{f}\right)\right)=1$ and $\models_{\left(\mathcal{M}, \mathfrak{T}_{\mathfrak{a}_{\mathfrak{t}}}\right)} \mathcal{K} \mathbf{a}_{t} \mathcal{K} \mathbf{b}_{t} \mathbf{f}$.

Notice that

$$
\vDash_{\left(\mathcal{M}, \mathfrak{T}_{\mathfrak{a}_{\mathfrak{t}}}\right)} \mathcal{K} \mathbf{a}_{t} \mathcal{K} \mathbf{b}_{t} \mathbf{f} \nRightarrow \vDash_{\left(\mathcal{M}, \mathfrak{T}_{\mathfrak{b}_{\mathfrak{t}}}\right)} \mathcal{K} \mathbf{b}_{t} \mathbf{f} .
$$

In other words, the following situation is possible:

- According to Alice's truth-perspective, it is true that Alice knows that Bob knows the Falsity.

- However, according to Bob's truth-perspective it is not true that Bob knows the Falsity.

Roughly, we might say: Alice knows that Bob is wrong. However, Bob is not aware of being wrong!

The epistemic situations illustrated above seem to reflect pretty well some characteristic limitations of the real processes of acquiring information and knowledge. Owing to the limits of epistemic domains, understanding and knowing are not generally closed under logical consequence. Hence, the unpleasant phenomenon of logical omniscience is here avoided. We have, in particular, that knowledge is not generally closed under logical conjunction, as in fact happens in the case of concrete memories both of human and of artificial intelligence. It is also admitted that an agent can understand (or know) a conjunction, without being able to understand (to know) its

members. Such situation, which might appear prima facie somewhat "irrational", seems to be instead deeply in agreement with our use of natural languages, where sometimes agents show to use correctly and to understand some global expressions without being able to understand their (meaningful) parts.

\section{REFERENCES}

[1] M.L. Dalla Chiara, H. Freytes, R. Giuntini, A. Ledda, R. Leporini, G. Sergioli, "Entanglement as a semantic resource", Foundations of Physics 40, pp. 1494-1518, 2011. 
[2] E. Beltrametti, M.L. Dalla Chiara, R. Giuntini,R. Leporini, G. Sergioli, "Epistemic Quantum Computational Structures in a Hilbert-Space Environment", Fundamenta Informaticae 115, pp. 1-14, 2012. DOI 10.3233/FI-2012-637.

[3] E. Beltrametti, M.L. Dalla Chiara, R. Giuntini, G. Sergioli, "Quantum teleportation and quantum epistemic semantics", Mathamatica Slovaca 62, pp. 1-24, 2012.

[4] G. Sergioli, R. Leporini, "Quantum approach to Epistemic Semantics", to appear in Soft Computing, Doi:10.1007/s00500-015-1781-7.

[5] G. Chiribella, G. M. D'Ariano, P. Perinotti, "Transforming quantum operations: Quantum supermaps", A Letters Journal Exploring the Frontiers of Physics 83, pp. 30004-p1-30004-p6, 2008.

[6] Hong-yi Fan, Li-yun $\mathrm{Hu}$, "Infinite-dimensional Kraus operators for describing amplitudue-damping channel and laser process", Optics Communications 282, pp. 932-935, 2009.

[7] K. Kraus, States, Effects and Operations, Springer, Berlin, 1983.

(Beltrametti) Dipartimento di Fisica, Università di Genova, Via Dodecaneso, 33, I-16146 Genova, Italy

E-mail address: enrico.beltrametti@ge.infn.it

(Dalla Chiara) Dipartimento di Filosofia, Università di Firenze, Via BologNESE 52, I-50139 FiRENZe, ITALY

E-mail address: dallachiara@unifi.it

(R. Giuntini) Dipartimento di Filosofia e Teoria delle Scienze Umane, Università di Cagliari, Via Is Mirrionis 1, I-09123 Cagliari, Italy.

E-mail address: giuntini@unica.it

(R. Leporini) Dipartimento Di Ingegneria, Universitì di Bergamo, Viale MarCONi 5, I-24044 Dalmine (BG), ItALy.

E-mail address: roberto.leporini@unibg.it

(G. Sergioli) Dipartimento di Filosofia e Teoria delle Scienze Umane, Università di Cagliari, Via Is Mirrionis 1, I-09123 Cagliari, Italy.

E-mail address: giuseppe.sergioli@gmail.com 